\title{
Characteristics of koala (Phascolarctos cinereus adustus) semen collected by artificial vagina
}

\author{
S. D. Johnston ${ }^{1 *}$, P. O'Callaghan ${ }^{2}$, M. R. McGowan ${ }^{1}$ and \\ N. J. Phillips ${ }^{1}$ \\ ${ }^{1}$ School of Veterinary Science, University of Queensland, 4072, Brisbane, Queensland, Australia; and \\ ${ }^{2}$ Lone Pine Koala Sanctuary, Jesmond Road, Fig Tree Pocket, 4069, Brisbane, Queensland, Australia
}

\begin{abstract}
Collection of semen using a modified ovine artificial vagina was attempted on 90 occasions from 25 Queensland koalas (Phascolarctos cinereus adustus). Complete ejaculates consisting of a copulatory plug and sperm fraction were collected on 36 occasions (40\%) from 19 animals. Seventeen of the males produced a complete ejaculate on their first or second service. Failure to collect semen $(38 \%)$ and the collection of partial ejaculates (14.5\%) was attributed to lack of sexual interest, aggressive behaviour towards the female by the male, the use of non-compliant teaser females or a distinct dislike of the artificial vagina. Only a few ejaculates were contaminated with urine $(4.5 \%)$ or obtained after ejaculation behaviour was terminated $(3 \%)$. The mean ( \pm SEM) values for the seminal characteristics of 19 complete ejaculates were: mass of copulatory plug fraction $0.78 \pm 0.10 \mathrm{~g}$, sperm fraction volume $0.73 \pm 0.10 \mathrm{ml}$, sperm concentration $165.1 \pm 26.7 \times 10^{6} \mathrm{ml}^{-1}, \mathrm{pH}$ of sperm fraction $6.7 \pm 0.2$, osmolarity of sperm fraction $315.0 \pm 5.4 \mathrm{mOsm}$, percentage forward motility $70.7 \pm 1.8 \%$, rate of sperm movement $4.0 \pm 0.1$ and percentage of abnormal spermatozoa $26.9 \pm 2.5 \%$. Percentages of sperm head morphotypes and tail abnormalities were documented. Although the artificial vagina technique is limited by the need for access to oestrous females, the procedure described has been shown to be a simple, reliable method of collecting semen from captive koalas.
\end{abstract}

\section{Introduction}

With the exception of recovery of epididymidal spermatozoa from necropsied specimens, electroejaculation is the most common method of collecting spermatozoa from marsupials, Tribe et al. (1994) and Taggart (1994) indicate that semen has been collected by electroejaculation from 12 species of marsupial representing five families (Phalangeridae, Peramelidae, Phascolartcidae, Vombatidae and Macropodidae). However, electroejaculation is an invasive technique requiring the animal to be anaesthetized to facilitate administration of a small electrical stimulus via a rectal probe. In addition, semen samples obtained by electroejaculation may not be representative of the natural ejaculate; they are typically greater in volume, with lower concentration of spermatozoa and higher $\mathrm{pH}$ (Watson, 1978).

In contrast, semen collection using an artificial vagina provides a non-invasive, reliable method for collecting natural ejaculates. The only disadvantage of this method is that it often requires intensive training of the male. To date the only report of the use of an artificial vagina to collect semen from a koala was that of Biery (1991). However, her description of the technique was brief and problems associated with semen manipulation after collection prevented description of the ejaculate characteristics.

Revised 24 July 1996.
This study reports a simple protocol for the collection of koala semen using a modified ovine artificial vagina. Ninety attempts to collect semen from a total of 25 Queensland koalas are documented. The seminal characteristics of ejaculates collected by artificial vagina from 19 koalas are recorded and represent the first published information for a marsupial.

\section{Materials and Methods}

\section{Animals}

Collection of semen using an artificial vagina was attempted on 90 occasions from 25 mature koalas in good body condition (Mode 9, Ellis and Carrick, 1992), at Lone Pine Koala Sanctuary (Brisbane, Queensland) from midSeptember 1995 to early February 1996. Mean ( \pm SEM and range) age (months), body weight $(\mathrm{kg})$ and testicular volume (ml) (Wildt et al., 1991) were 69.4 $\pm 5.0(n=25), 7.8 \pm 0.1$ $(n=24)$ and $4.45 \pm 0.13(n=19)$, respectively. Koalas were housed in semi-enclosed cages furnished with a network of poles and branches and were fed fresh eucalyptus leaf ad libitum once a day (Blanshard, 1994). Males and females were housed separately in groups of eight to ten per enclosure. Only nine of the 25 male koalas used in this study had previously been used for mating. 


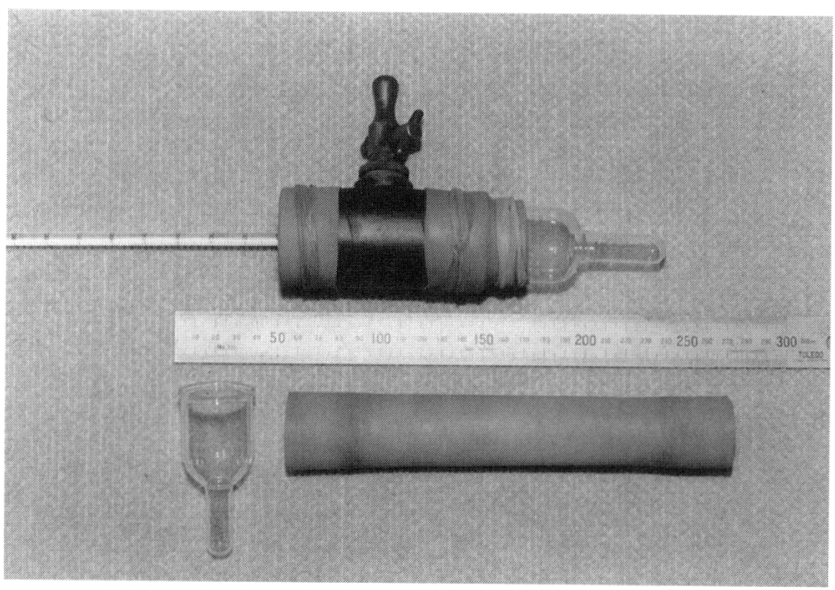

Fig. 1. Koala (Phascolarctos cinereus) artificial vagina. (Body: length $85 \mathrm{~mm}$, outer diameter $47 \mathrm{~mm}$, inner diameter $38 \mathrm{~mm}$. Liner: length $150 \mathrm{~mm}$, outer diameter $37 \mathrm{~mm}$, thickness $0.5 \mathrm{~mm}$ ).

\section{The artificial vagina}

A standard ovine artificial vagina (Watson, 1978) with an inner diameter of $38 \mathrm{~mm}$ and outer diameter of $47 \mathrm{~mm}$ was cut down to a length of $85 \mathrm{~mm}$ to accommodate the length of the koala penis (Fig. 1). A $0.5 \mathrm{~mm}$ thick flexible soft rubber liner with an outer diameter of $37 \mathrm{~mm}$ was used as a liner. The artificial vagina was initially filled with $25-30 \mathrm{ml}$ of hot water $\left(65^{\circ} \mathrm{C}\right)$ and then inflated with air. The open end of the artificial vagina was lubricated with $K-Y$ jelly. The internal temperature of the artificial vagina immediately before use was approximately $43^{\circ} \mathrm{C}$.

\section{Collection procedure}

Before collection, a sexually mature teaser male was brought into an enclosure housing between 10 and 12 sexually mature females. The presence of the male facilitated detection of behavioural oestrus (Blanshard, 1994). A female koala displaying behavioural oestrus was placed on a vertical tree pole approximately $1.5 \mathrm{~m}$ from the ground. The male koala from which semen was to be collected was placed immediately below the female and allowed to establish a copulatory position (Smith, 1980). The male would secure this position restraining the female by means of a neck bite to the nape of her neck. The male was allowed to achieve intromission and thrust his penis into the female's urogenital sinus five to ten times before the penis was diverted into the artificial vagina, which was held initially in a near vertical position. Penile thrusting into the artificial vagina continued for approximately $30 \mathrm{~s}$, culminating in one or two final vigorous ejaculatory thrusts. Once penile thrusting had ceased, the artificial vagina was directed downward to just below a horizontal position to allow the ejaculated semen to flow into the collection vial. After approximately $45 \mathrm{~s}$, the male would terminate copulation by means of a bite to the female's shoulder region. An aggressive interaction followed and the pair would separate. During separation the artificial vagina remained held over the penis but the pressure in the artificial vagina was released by opening the valve. This procedure was found necessary as three koalas ejaculated a sperm fraction after ejaculation behaviour had ceased. The period of copulation, defined as the time between the initial neck bite and final shoulder bite, was recorded on video tape for 16 collections by artificial vagina. Similarly the periods of penile thrusting and ejaculation were also recorded for these collections.

\section{Ejaculate type and collection schedule}

Two types of ejaculate were collected: complete and partial ejaculates. The complete ejaculate contained two distinct fractions of seminal fluid. One of these fractions immediately solidified within the collection vial or in the liner of the artificial vagina into a white rubbery mass containing few or no spermatozoa. The volume of this copulatory plug fraction was estimated by weighing the solidified mass. The volume of the other fraction was also determined. This fraction was khaki to creamy in colour and contained motile spermatozoa. A few of the ejaculates were not distinctly fractionated, typically milky white in colour and contained few spermatozoa. These were considered partial ejaculates and their seminal characteristics were not evaluated.

Semen collection was repeatedly attempted from each male to obtain at least two complete ejaculates per koala. While some males required only two collections to produce their quota, other males required up to eight collections or produced only one complete ejaculate. Six koalas failed to produce a complete ejaculate. Complete ejaculates were collected from 19 males, 15 of which were replicate samples.

\section{Seminal characteristics of the ejaculate}

The $\mathrm{pH}$ of the sperm fraction was determined using narrow range $\mathrm{pH}$ paper strips $( \pm 0.3$ ) (Prolabo, Paris) and osmolarity evaluated using a vapour pressure osmometer (Wescor, Utah). Sperm concentration (sperm $\times 10^{6} \mathrm{ml}^{-1}$ ) was estimated using a Makler counting chamber (Makler Inc., Israel). Motility of spermatozoa was assessed by diluting $50 \mu \mathrm{l}$ of sperm fraction 1:4 with PBS (Commonwealth Serum Laboratories, Brisbane) containing no $\mathrm{Mg}^{2+}$ or $\mathrm{Ca}^{2+}$. The percentages of both forward motile and circular motile spermatozoa were recorded using phase-contrast microscopy $(\times 100)$ equipped with a warm stage set at $35^{\circ} \mathrm{C}$. The rate of sperm movement was determined using the criteria of Barth (1995). Nigrosin eosin stained smears (Barth and Oko, 1986) were prepared to evaluate sperm morphology. The percentage individual head morphotypes and sperm abnormalities were calculated for each ejaculate. Descriptions of abnormal spermatozoa have been described by Johnston et al. (1994).

\section{Statistical analysis}

Correlation between age, testicular volume and all seminal characteristics from the 19 koalas that produced complete ejaculates were calculated. Similarly, all seminal characters (including sperm head morphotypes and abnormalities) were correlated with each other to determine any intra-seminal relationships. 


\section{Results}

When collecting semen with the artificial vagina the mean copulation time for 16 koalas was $74.7 \pm 3.2 \mathrm{~s}$, consisting of a $27 \pm 2.1 \mathrm{~s}$ penile thrusting period, followed by a $44.8 \pm 3.2 \mathrm{~s}$ period of ejaculation. Overall, semen collection was attempted on a total of 90 occasions. On 28 occasions (31\%) 14 males failed to display any sexual interest in the femaie. On only six of these occasions was lack of libido associated with the presentation of non-compliant females that would not 'stand' for the full length of copulation. On 12 of 28 occasions, lack of male sexual interest was associated with aggressive behaviour by the male towards the female. In all these cases the male chose to bite the female on her elbow rather than attempt service.

When the artificial vagina was used with males exhibiting sexual interest, complete ejaculates were recovered on 36 of 62 attempts $(58 \%)$ from a total of 19 males. Four of the $62(6 \%)$ attempts resulted in semen being contaminated with urine. On 13 occasions (21\%) partial ejaculates were recovered; three were associated with collections using non-compliant females and three were from males that initially exhibited good libido and normal mating behaviour but subsequently stopped or reduced penile thrusting when the artificial vagina was placed over the penis. On three occasions (5\%) semen was ejaculated after, or during the removal of the artificial vagina from the penis. Six $(10 \%)$ artificial vagina collection attempts produced no semen, despite males showing sexual interest. Three of these attempts were associated with collections using noncompliant females, while the other three were associated with a complete cessation or a reduction in penile thrusting behaviour following the application of the artificial vagina.

Complete ejaculates were collected from 12 of the 25 males on their first attempt of using the artificial vagina. Six of the 12 had never been mated before. A further five koalas required two attempts, while another two required three services to produce their first complete ejaculate. For four of the six koalas that failed to produce an ejaculate, semen collection was attempted only once.

The mass of the copulatory plug fraction and the seminal characteristics of the sperm fraction of ejaculates from 19 koalas collected by artificial vagina are presented (Table 1). Records of mean ( \pm SEM) percentage sperm head morphotypes and abnormalities from the same 19 koalas are shown in Table 2 .

There was a significant relationship $(P=0.047 ; r=+0.46)$ between koala age and testicular volume, but not between koala weight and testicular volume. In this study there was no correlation of any combination of age, weight or testicular volume when compared with any seminal characteristic. However, percentage forward motility was found to be significantly correlated with the percentage of total sperm abnormalities $(P=0.040 ; r=-0.48)$, the percentage of broken necked spermatozoa $(P=0.042 ; r=-0.47)$ and the percentage of simple bent midpieces $(P=0.019 ; r=-0.53)$. There was also a non-significant trend between forward motility and osmolarity of the ejaculate $(P=0.057 ; r=-0.46)$. The apparent negative effect of mid-piece abnormalities on the percentage of forward motility was confirmed by demonstrating a positive correlation between circular motility and the percentage of total abnormal spermatozoa $(P=0.003 ; r=0.45)$, broken necked spermatozoa
Table 1. Mass of plug fraction (mean \pm SEM) and seminal characteristics from the sperm fraction collected by artificial vagina from 19 koalas

\begin{tabular}{lr} 
Seminal characteristic & Mean \pm SEM \\
\hline Plug fraction mass $(\mathrm{g})$ & $0.78 \pm 0.1$ \\
Sperm fraction volume $(\mathrm{ml})$ & $0.73 \pm 0.1$ \\
Sperm concentration $\left(\times 10^{6} \mathrm{ml}^{-1}\right)$ & $165.1 \pm 26.7$ \\
Total sperm in the ejaculate $\left(\times 10^{\circ}\right)$ & $107.9 \pm 16.1$ \\
pH of sperm fraction $( \pm 0.3)$ & $6.7 \pm 0.2$ \\
Osmolarity of sperm fraction (mOsm) & $315.0 \pm 5.4$ \\
Percentage of forward motile spermatozoa & $70.7 \pm 1.8$ \\
Percentage of circular motile spermatozoa & $9.0 \pm 1.4$ \\
Rate of sperm movement $(0-5)$ & $4.0 \pm 0.1$ \\
Percentage of abnormal spermatozoa & $26.9 \pm 2.5$
\end{tabular}

Table 2. Head morphotypes and sperm abnormalities (mean \pm SEM) found in the ejaculates collected by artificial vagina from 19 koalas

\begin{tabular}{lr}
\hline Head morphotypes & Mean \pm SEM \\
\hline I & $17.2 \pm 1.8$ \\
II & $15.3 \pm 2.3$ \\
III & $26.8 \pm 1.9$ \\
IV & $10.6 \pm 0.9$ \\
V & $2.8 \pm 1.1$ \\
VI & $2.8 \pm 0.5$ \\
VIl & $9.3 \pm 1.2$ \\
VIII & $1.8 \pm 0.6$ \\
IX & $2.5 \pm 0.4$ \\
X & $1.2 \pm 0.3$ \\
XI & $2.4 \pm 0.3$ \\
T & $7.3 \pm 1.3$ \\
Sperm abnormalities & \\
'Broken-neck' & $17.9 \pm 2.3$ \\
Bent mid-piece & $4.4 \pm 0.6$ \\
Loose heads & $2.9 \pm 1.3$ \\
Segmental aplasia of the mid-piece & $0.9 \pm 0.3$ \\
Multiple tails & $0.3 \pm 0.2$ \\
\hline
\end{tabular}

$(P=0.008 ; r=+0.59)$ and the percentage of spermatozoa with simple bent midpieces $(P=0.049 ; r=+0.46)$.

\section{Discussion}

While the period of thrusting behaviour reported in this study was similar to that of natural copulation (Smith, 1980), the interval from ejaculation to disengagement was substantially longer. In this study there was clear delineation between penile thrusting and ejaculation behaviour. Penile thrusting stopped after two final distinct ejaculatory thrusts. On the final thrust the male pushed his pelvis hard up against the artificial vagina by arching his back and maintaining this position for the rest of the copulatory act. 
The success of artificial vagina semen collection using the artificial vagina in the koala is likely to be related to the reproductive behaviour and strong sexual drive of the animal. Once the male has established a copulatory position, the erect penis is repeatedly thrusted in the direction of the urogenital opening. This behaviour combined with the male's maintenance of the neck bite, allow the collector to manipulate the penis readily into the artificial vagina without any interference by the male.

One of the most important technical considerations of the collection by artificial vagina is the manipulation of the penis and artificial vagina into a downward position. Excessive bending of the penis may only serve to constrict urethral flow and possibly cause injury to the penis. It is possible to overcome the problem of semen flow by tilting the rump of the male slightly upwards during the collection procedure.

Clearly, a major limitation of the artificial vagina procedure is its reliance on male libido. There is obviously still much to understand about the mating system of intensively managed captive koala populations. It is likely that dominance relationships, territoriality, receptivity of the female and copulation frequency are important variables in interpreting the lack of sexual response by males.

Complete ejaculates were never recovered from males when non-compliant teaser females were used. In contrast, females that are fully in oestrus, typically 'stand' still for the male during the mating procedure, only struggling free from the male when a bite is applied to the shoulder region. The need for females in oestrus may be a potential limitation for the use of the artificial vagina semen collection on a routine basis.

During six collection attempts some males appeared to either reduce the rate of their penile thrusting behaviour or to stop completely. The temperature of the artificial vagina was possibly too low on two of these occasions and too high on another. Urine contamination during semen collection occurred on four occasions; twice from one individual. Usually on initial presentation of a male into a female enclosure, the male moves excitedly about the enclosure scent marking poles or walls with his sternal gland and urinating. On at least two occasions of urine contamination after ejaculation, the associated males failed to urinate on initial presentation.

Semen collection by artificial vagina usually involves training of animals for service (Watson, 1978). The majority of koalas in this study required little if any training. Within two attempts, 17 animals had produced a complete ejaculate.

In this study, semen collected by artificial vagina was ejaculated in two distinct fractions. A white non-spermic fluid was usually collected first and coagulated quickly, either in the collection vessel, where it tended to settle onto the bottom of the vial, or into the liner of the artificial vagina. A second fraction, khaki in colour, usually contained large numbers of spermatozoa. If ejaculation into the artificial vagina is similar to that of natural ejaculation, the sperm fraction must be deposited and trapped behind that of the plug material. While this appears an ideal situation for the retention of semen in the urogenital to lower-vaginal complex following coitus in an arboreal species, further observations are required to confirm this unusual mechanism of copulatory plug formation.
Although uncommon, Wildt et al. (1991) and Johnston et al. (1994) have both reported coagulation of koala electroejaculates. Biery (1991) in her original description of collection by artificial vagina in the koala also indicated that koala semen samples coagulated to form a solid plug without warning. In this study coagulation of a partial ejaculate occurred only twice and from the same koala.

Watson (1978) has commented that semen collected by artificial vagina is characteristically lower in $\mathrm{pH}$ than electroejaculated semen. Wildt et al. (1991) and Johnston et al.(1994) reported the mean $\mathrm{pH}$ of electroejaculated semen as 6.1 $(n=47)$ and $6.9(n=8)$, respectively. The mean $\mathrm{pH}$ of 36 natural ejaculates from 19 koalas in this study was 6.7 .

There appears to be no published data on the osmolarity of ejaculated semen in marsupials. In koalas, spermatozoa are ejaculated in seminal fluid slightly hypertonic to physiological saline $(290 \mathrm{mOsm})$. While not statistically significant, forward motility was found to be negatively associated with osmolarity $(P=0.057)$. Although further investigation is required to establish a significant causal relationship, this finding may be useful in developing extension and freezing diluents for koala semen.

Forward motility and rate of movement for spermatozoa collected by artificial vagina were similar to those of electroejaculated spermatozoa (Wildt ef al., 1991; Johnston et al. 1994). Forward motility was found to be negatively correlated with the percentage of broken-neck (Temple-Smith and Taggart, 1990) and simple bent mid-piece sperm abnormalities. This is perhaps the first evidence in the marsupial that demonstrates that sperm abnormalities may have a significant effect on sperm motility.

Although the proportions of head morphotypes described in this study were not directly compared with those of Wildt et al. (1991) and Johnston et al. (1994), some general observations can be noted. First, there appear to be four to five distinct head types that occur in greater proportion than others (I, II, III, IV and VII). Secondly, the presence of type XI and teratoid sperm heads not reported by Wildt et al. (1991) but described by Johnston et al. (1994) were confirmed in the ejaculates of all koalas analysed in this study. Given that all koalas sampled where considered to be in good body condition and disease free, the percentage of sperm abnormalities (26.9\%) reported in this study is likely to be a valid representation of the situation in normal ejaculates.

In conclusion, the koala artificial vagina procedure satisfies all the criteria of an ideal semen collection technique described by Watson (1978). Although the technique is somewhat limited by the need for numerous oestrous females, the procedure offers great promise for the regular and non-invasive collection of koala semen. However, it is unlikely to replace electroejaculation for semen collection from koalas in free-range and small captive populations.

The authors would like to thank the staff of Lone Pine Koala Sanctuary for their assistance in koala handling and video recording of the semen collection procedure.

\section{References}

Barth AD (1995) Evaluation of frozen bovine semen by the veterinary practitioner Proceedings of Bovine Short Course pp 105-110 American College of Theriogenologists and Society for Theriogenology, Hastings, New England 
Barth AD and Oko RJ (1986) Abnormal Morphology of Bovine Spermatozoa lowa State University Press, lowa

Biery K (1991) Sex and the single koala Zoonooz 64 12-13

Blanshard WH (1994) Medicine and husbandry of koalas. In The TG Hungerford Refresher Course for Veterinarians Proceedings 233 pp 547-627 Post-Graduate Committee of Veterinary Science, University of Sydney

Ellis WAH and Carrick FN (1992) Total body water and the estimation of fat in the koala (Phascolarctos cinereus) Australian Veterinary Journal 69 229-230

Johnston SD, McGowan MR, Carrick FN, Cameron RDA and Tribe A (1994) Seminal characteristics and spermatozoal morphology of captive Queensland koalas (Phascolarctos cinereus adustus) Theriogenology 42 501-511

Smith M (1980) Behaviour of the koala Phascolarctos cinereus (Goldfuss) in captivity V*: sexual behaviour Australian Wildlife Research 7 41-51

Taggart DA (1994) A comparison of sperm and embryo transport in the female reproductive tract of marsupial and eutherian mammals Reproductive Fertility and Development 6 451-472
Temple-Smith PD and Taggart DA (1990) On the male generative organs of the koala (Phascolarctos cinereus) an update. In Biology of the Koala pp 33-54 Eds AK Lee, LA Handasyde and GD Sanson. Surrey Beatty and Sons Pty Ltd, Sydney

Tribe A, Johnston SD and Carrick FN (1994) Artificial reproduction in marsupials. In The TG Hungerford Refresher Course for Veterinarians Proceedings 233 pp 71-109 Post-Graduate Committee of Veterinary Science, University of Sydney

Watson PF (1978) A review of techniques of semen collection in mammals Symposium of the Zoological Society of London 43 97-126

Wildt DE, Bush M, O'Brien SJ, Murray ND, Taylor A and Marshall-Graves JA (1991) Semen characteristics in free-living koalas (Phascolarctos cinereus) Journal of Reproduction and Fertility 92 99-107 